\title{
OUTCOMES OF THE 2015 WORLD RADIOCOMMUNICATION CONFERENCE FOR AERONAUTICAL SPECTRUM AND APPLICATIONS
}

\author{
Robert J Kerczewski, NASA Glenn Research Center, Cleveland, Ohio, USA \\ Loftur Jonasson, International Civil Aviation Organization, Montreal, Canada
}

\begin{abstract}
At the conclusion of a nearly four year study cycle following the closing of the 2012 World Radiocommunication Conference (WRC-12), the 2015 WRC in November of 2015 considered a number of agenda items and issues relevant to systems and spectrum allocations supporting communications, navigation and surveillance for the operation of civil aviation. Among a number of WRC-15 agenda items and issues, the key agenda items affecting civil aviation included: unmanned aircraft systems use of the Fixed Satellite Service for command and control communications; global flight tracking; new allocations to International Mobile Telecommunications (IMT); and protection of the Fixed Satellite Service to support safe operation of aircraft. A number of other agenda items affecting or potentially affecting civil aviation were also addressed by WRC-15. In this paper we describe the outcomes of WRC-15 for these civil aeronauticalrelevant issues. We then outline the civil aviationrelated agenda items and issues that will be considered at the upcoming 2019 WRC.
\end{abstract}

\section{Introduction}

The International Telecommunications Union Radiocommunication Sector (ITU-R) is charged with regulating the use of the radiofrequency spectrum, enabling the optimum use of this very limited resource in a way which provides for all needs and requirements as efficiently as possible while minimizing interference and other disruptions to the operations of systems and equipment making use of spectrum. One of the primary ways this is accomplished is through quadrennial World Radiocommunication Conferences (WRC), during which changes to the allocation of spectrum to various services, and associated regulations governing the operation of those services, is agreed upon by ITU member administrations (ITU is a specialized agency of the United Nations).
The WRC acts on agenda items approved by resolution at the previous WRC which generally involve adding or modifying allocation of spectrum between services. In addition, revisions of the regulations governing use of radiofrequency spectrum are considered.

The ITU-R defines more than 30 radiocommunication services to which spectrum can be allocated, for example: Fixed Satellite Service, Land Mobile Service, Maritime Mobile Service, Broadcasting Service, Radionavigation Service, Earth Exploration Satellite Service, Space Research Service, etc.

Several of these services are specific to aeronautical use, including: Aeronautical Mobile Service, Aeronautical Mobile (Route) Service, Aeronautical Mobile Satellite Service, Aeronautical Mobile Satellite (Route) Service, Aeronautical Radionavigation Service, and Aeronautical Radionavigation Satellite Service.

Demand for radiofrequency spectrum to support the growth of existing applications and emerging new applications and services puts tremendous pressure on existing applications and services. For example, International Mobile Telecommunications (IMT), representing the cellular and mobile telecommunications industry, is constantly seeking new spectrum to support emerging generations of mobile telephony (i.e. LTE, 4G, 5G, etc.) with ever increasing bandwidth requirements. Unmanned aircraft systems (UAS) is an emerging but rapidly expanding new technology requiring new spectrum allocations to support command and control links, detect and avoid systems, and payload communication.

Civil aviation in general represents an existing technology supported by existing spectrum allocations to aeronautical services, and is being pressured by the emerging and expanding services in terms of spectrum available to support its operations. 
At the same time, the growth of civil aviation creates more spectrum users and more complex air traffic management requirements, leading to more intense spectrum usage. Civil aviation also has emerging new technologies and applications requiring access to spectrum, UAS being a prime example.

As a result, the WRC has many agenda items and regulatory actions that impact civil aviation. The International Civil Aviation Organization (ICAO), itself a United Nations specialized agency, has a leading role in addressing WRC agenda items and regulatory actions affecting civil aviation, through development and advocacy of a position on WRC agenda items developed for each WRC.

In addition, many administrations develop position statements on aviation-related agenda items, receiving technical support from their national aviation and technical agencies and interested industries and other organizations. For example, in the United States, the US State Department leads the US delegation at the WRC, supported by such agencies as the Federal Aviation Administration, the National Telecommunications and Information Administration, the Federal Communications Commission, the National Aeronautics and Space Administration, and the Department of Defense. Major corporations and industry and trade associations also provide technical support to the WRC process for issues of importance to their industries. The same is true to varying degrees for many other nations with significant aviation industries and interests.

Thus, a significant level of activity is undertaken for the civil aviation-related issues and agenda items at the WRC.

Agenda items affecting civil aviation interests fall into three general categories. The first category involves changes in spectrum allocations directly affecting operation of civil aviation systems. In these cases, new allocations or changes to existing allocations are considered for frequency bands under which civil aviation systems operate. The second category involves changes in spectrum allocations or regulations indirectly affecting operation of civil aviation systems. For example, changes to allocations in a band adjacent to a band under which civil aviation systems operate which may impact those systems through interference, operational limitations, or other effects. The third category involves regulatory changes which do not directly involve changes to spectrum allocations but may still have an impact on civil aviation systems, such as changes in definition of system characteristics that could, for example, impact compatibility between aviation systems and non-aviation systems.

The 2015 WRC (WRC-15), held from 2 to 27 November 2015 in Geneva, Switzerland, included a number of issues and agenda items of importance to civil aviation, resulting in an intense and spirited debate among the approximately 3800 delegates from 162 ITU Member States and 130 other entities participating in the conference. Although issues related to all three categories described above were addressed at WRC-15, this paper focuses on civil aviation-related WRC-15 agenda items. The following section describes the agenda items and the outcomes for four key agenda items, based primarily on the report by the ICAO Frequency Spectrum Management Panel Secretary on the results of WRC15; this report contains many additional details and information [1]. A brief description is then provided for the outcomes of several other civil aviationrelated agenda items. An initial review of civil aviation-related issues and agenda items for the next WRC, scheduled for 2019, is provided.

\section{WRC-15 Key Civil Aviation Outcomes}

More than 30 agenda items were considered by WRC-15 [2]. Of these, 4 were of significant importance to civil aviation. These include Agenda Items 1.1, 1.5, 9.1.5 and Global Flight Tracking.

The outcomes for these agenda items are generally considered to be favorable to civil aviation interests. The resolutions adopted by WRC-15 are published in the WRC-15 Final Acts [3]. The following subsections describe the agenda items and the outcomes for the four key civil aviation agenda items.

\section{WRC-15 Agenda Item 1.1 - International Mobile Telecommunications (IMT)}

Agenda Item $1.1 \quad$ (AI 1.1) considered "additional spectrum allocations to the mobile service on a primary basis and identification of additional frequency bands for International Mobile Telecommunications (IMT) and related regulatory provisions, to facilitate the development of terrestrial mobile broadband applications..." 
The demand for spectrum for mobile and broadband applications is growing at a fast pace. Under AI 1.1, the telecommunications industry was seeking up to $1200 \mathrm{MHz}$ of additional spectrum in the $300 \mathrm{MHz}$ to $6 \mathrm{GHz}$ range for IMT. This frequency range includes a number of aeronautical frequency bands and several of these were examined by the conference for a potential allocation to IMT, including 2700-2900 MHz used by aeronautical primary surveillance radar (PSR) and 4.4-4.5 GHz which is adjacent to the aeronautical radio altimeter band at 4.2-4.4 GHz. The aeronautical radio altimeters constitute a critical component of the aircraft ground proximity warning system and are used during CAT I/II/III landings.

While studies in the ITU prior to the conference had indicated that sharing is not possible between PSR and IMT, the ITU had performed no studies to analyze adjacent band compatibility between IMT and the radio altimeters. ICAO studies under the Frequency Spectrum Management Panel (FSMP) prior to the conference indicated a strong potential for harmful interference to the radio altimeters by IMT. Relatively early during the conference an agreement was reached to take PSR bands out of consideration. However, 4.4-4.5 GHz, adjacent to the radio altimeter band was not off the table until the final days of the conference due to actions by ICAO, the International Air Transport Association (IATA) and other aeronautical stakeholders during the conference. As a result, WRC-15 made no IMT allocations impacting the PSR band or radio altimeter operations.

In addition, a favorable outcome was secured for the C-band (3.4-4.2 GHz), used for satellite links in the Fixed Satellite Service (FSS). Such links are used to support aeronautical ground-ground communications, using very small aperture terminal (VSAT) equipment, especially in Africa and South and Central America. The conference made no new allocations or identifications for IMT between 3.7 and $4.2 \mathrm{GHz}$. In the remainder of the C-band (less critical for aviation VSATs), the conference specified necessary conditions to protect the FSS.

\section{WRC-15 Agenda Item 1.5 - Unmanned Aircraft Systems}

Agenda Item 1.5 (AI 1.5) considered "the use of frequency bands allocated to the fixed-satellite service not subject to Appendices 30, 30A and 30B for the control and non-payload communications of unmanned aircraft systems (UAS) in non-segregated airspaces..."

Previous World Radiocommunication Conferences have now considered allocations for both line-of-sight and beyond line-of-sight communication links for the Command and Control (C2) of Unmanned Aircraft Systems (UAS), with allocations suitable for line-of-sight operations being obtained at WRC-12. Existing satellite networks operating in the fixed satellite service (FSS) in the frequency bands at 14/12 GHz (Ku-band) and 30/20 $\mathrm{GHz}$ (Ka-band) have potential spectrum capacity to meet the requirements for beyond line-of-sight communication links and could be used for UAS C2. WRC-12 had attempted but failed to develop a solution supporting the use of FSS spectrum for UAS in a manner satisfying the safety and regularity of flight requirements of civil aviation.

ICAO has determined that $\mathrm{C} 2$ links are aviation safety links and are required to be operated using aviation safety spectrum. However, the FSS is normally not recognized in the ITU as a safety service. Although state-operated UAS have been using C2 links over FSS in segregated airspace for a number of years, for non-segregated airspace additional conditions are needed to meet an equivalent level of safety. ICAO identified the required conditions in the ICAO Position for WRC15.

Taking into account the ICAO conditions, WRC-15 was able to come to an agreement to make new allocations in the FSS $\mathrm{Ku}$ and $\mathrm{Ka}$ frequency bands, identifying over $2.2 \mathrm{GHz}$ of spectrum in WRC-15 Resolution 155. The Resolution specifies that these frequency bands can be used for the UAS C2 links in non-segregated airspace and any other airspace under the control of civil aviation authorities. This use is contingent on the successful development of ICAO Standards and Recommended Practices (SARPs). The Resolution goes into considerable detail to protect the current FSS environment against being disrupted by the introduction of a safety service. While not providing a traditional aeronautical mobile satellite (route) service (AMS(R)S), the Resolution introduces an application with safety of life implications into the FSS and contains language consistent with a safety 
service and appropriately referencing article 4.10 of the Radio Regulations. While ensuring the highest degree of certainty against harmful interference, to the extent possible under the conditions governing the coordination process for FSS, this approach also takes care of the moral obligation by States and spectrum users for safeguarding life in the use of the relevant frequencies. However, a part of the burden to ensure operation without harmful interference, such as real time interference monitoring and predicting interference risks, will have to be addressed in SARPs and is thus placed on the shoulders of the aeronautical regulators. Further, the Resolution requires ICAO to report on its progress in the development of SARPs for the UAS C2 link to WRC-19 and WRC-23, including identification of any problems in the application of the Resolution and potential means by the WRC to address those. The Resolution comes fully into force by WRC-23.

Resolution 155 was achieved as the result of a difficult and delicate compromise by the conference and is considered by ICAO to be fully in line with the ICAO Position. It also provides ICAO's Remotely Piloted Aircraft Systems Panel (RPASP) with a set of conditions against which SARPs can be developed or to identify any impediments to the safe use of FSS for beyond line-of-sight UAS C2, if any. However, the implicit obligation of the ICAO work program to fully support the required ITU studies, to enable WRC-19 and WRC-23 to fine tune the Resolution before it enters into full force in 2023, should be noted. It is expected that this work will take place within the FSMP, RPASP and other supporting panels of ICAO's Air Navigation Commission.

\section{WRC-15 Agenda Item 9.1.5 - FSS Earth Stations for Safe Operation of Aircraft}

Agenda Item 9.1.5 considered "technical and regulatory actions in order to support existing and future operation of fixed-satellite service earth stations within the band 3 400-4 $200 \mathrm{MHz}$, as an aid to the safe operation of aircraft and reliable distribution of meteorological information in some countries in Region 1."

The efficient and safe provision of air navigation services requires the implementation and operation of ground communications infrastructure with high availability, reliability and integrity. In the Africa and the Indian Ocean Region, the difficulty of fulfilling these requirements, given the extent of the airspace and weakness in terrestrial communication infrastructure, led the ICAO AFI Planning and Implementation Regional Group in 1997 to approve the use of VSAT satellite terminals operating under the FSS to support terrestrial aeronautical communications services in the frequency range 3.4$4.2 \mathrm{GHz}$, a frequency range which remains the only viable option for satellite links with high availability in tropical regions, due to the pronounced rain attenuation at higher frequency bands.

While the frequency band $3.4-3.6 \mathrm{GHz}$ is allocated to the FSS, it is also allocated to the mobile service, identified for international mobile telecommunications (IMT). Aeronautical VSAT operations have experienced a number of cases of harmful interference due to lack of sufficient measures to protect them by administrations when licensing IMT or other services to co-share the band. To address this issue, the conference modified Resolution 154 to underscore the protection needs of existing and planned aeronautical and meteorological VSAT stations. The modified Resolution also highlights the need to license the VSAT stations and register them in the Master International Frequency Register (MIFR), to ensure that they are visible to all administrations concerned.

The modification to Resolution 154 developed by the conference is considered by ICAO to be fully in line with the ICAO Position. As a follow up measure, aviation service providers operating VSAT stations should be encouraged to have their stations licensed and registered in the MIFR. It is expected that the same measures will help ameliorate similar issues, currently experienced with the operation of aeronautical VSAT networks in South and Central America

\section{WRC-15 Agenda Item on Global Flight Tracking for Civil Aviation and a Future Agenda Item on the Global Aeronautical Distress and Safety System}

The Global Flight Tracking agenda item was established by the ITU 2014 Plenipotentiary Conference, under Resolution COM 5/1 which resolved "to instruct WRC-15, pursuant to No. 119 of the ITU Convention, to include in its agenda, as a matter of urgency, the 
consideration of global flight tracking, including, if appropriate, and consistent with ITU practices, various aspects of the matter, taking into account ITU-R studies" [4].

While the year 2014 represented one of aviation's safest years in terms of the number of accidents, the tragedy of Malaysia Airlines flight 370 in March 2014 highlighted vulnerabilities in the global air navigation system requiring urgent mitigation. To address these vulnerabilities, the aviation community embarked in a global effort to develop and implement a Global Aeronautical Distress and Safety System (GADSS), and forged consensus among its Member States and the international air transport industry sector on the nearterm priority to track airline flights, no matter their global location or destination. The ICAO Second High-level Safety Conference 2015 (HLSC 2015) supported that ICAO should encourage States and the ITU to discuss allocation requirements at WRC-15 to provide the necessary frequency spectrum allocations to enable global air traffic services (ATS) surveillance. The HLSC 2015 also endorsed a concept of operations to support future development of the GADSS. It is envisaged that GADSS will address issues such as: aircraft tracking under normal and abnormal conditions; autonomous distress tracking; automatic deployable flight recorder; and procedures and information management.

Addressing the issue of global flight tracking, WRC-15 agreed to an allocation to AMS(R)S (Earthto-space) at $1090 \mathrm{MHz}$, limited to the space station reception of Automatic Dependent SurveillanceBroadcast (ADS-B) emissions from aircraft transmitters operating in accordance with ICAO SARPs. The new allocation has to protect the aeronautical radionavigation service under which the aircraft secondary surveillance radar transponders and distance measuring equipment operate. The ITU$\mathrm{R}$ has been tasked to complete studies related to the space station reception of ADS-B, including consideration of potential interference from nonICAO standardized systems, and to report their outcome to ICAO. ICAO considers that this result fully accommodates the ICAO Position.

The conference further decided on an agenda item for WRC-2019, to consider spectrum needs and regulatory provisions for the introduction and use of GADSS. ICAO is invited to participate actively in the studies by providing requirements and information that should be taken into account in the studies of ITU-R, in particular the quantification and characterization of radiocommunication requirements related to the GADSS.

\section{Other WRC-15 Outcomes Relevant to Civil Aviation}

Several other WRC-15 agenda items are relevant to civil aviation, including agenda items $1.4,1.6,1.7$, $1.10,1.11,1.12,1.16,1.17,1.18,9.1 .1$ and 9.1.6. In the following subsections we provide a brief description of these agenda items and their outcomes.

\section{WRC-15 Agenda Item 1.4 - Amateur Service Secondary Allocation 5250-5450 MHz}

This agenda item considered "possible new allocation to the amateur service on a secondary basis within the band 5 250-5 $450 \mathrm{kHz} . . . "$ The ICAO Position on this agenda item was to ensure that any allocation made to the amateur service would not cause harmful interference to aeronautical systems operating under the allocation to the aeronautical mobile (route) service $(\mathrm{AM}(\mathrm{R}) \mathrm{S})$ in the adjacent $5450-5480 \mathrm{kHz}$ in ITU Region 2. The conference allocated $5351.5-5366.5 \mathrm{kHz}$ to the amateur radio service, limited to a maximum radiated power of below 25W. The new allocation provides a guard band of $83.5 \mathrm{kHz}$ thus fully protecting the aeronautical mobile (R) service.

\section{WRC-15 Agenda Item 1.6 - Primary Allocations to the FSS the range between 10 $\mathrm{GHz}$ and $17 \mathrm{GHz}$}

This agenda item considered "possible primary allocations to the fixed-satellite service (Earth-tospace and space-to-Earth) of $250 \mathrm{MHz}$ in the range between $10 \mathrm{GHz}$ and $17 \mathrm{GHz}$ in Region 1, and, possible primary allocations to the fixed-satellite service (Earth-to-space) of $250 \mathrm{MHz}$ in Region 2 and $300 \mathrm{MHz}$ in Region 3 within the range 13-17 GHz..." The ICAO Position on this agenda item was to oppose any new FSS allocation within the frequency bands $13.25-13.4$ and $15.4-15.7 \mathrm{GHz}$, which contain allocations for the aeronautical radionavigation service. The conference agreed on allocations outside of those of concern to civil aviation as noted in the ICAO Position. 
WRC-15 Agenda Item 1.7 - Review of the band 5 091-5 $150 \mathrm{MHz}$ by the FSS

This agenda item reviewed "the use of the band $5091-5150 \mathrm{MHz}$ by the fixed-satellite service (Earthto-space) (limited to feeder links of the nongeostationary mobile-satellite systems in the mobilesatellite service)..." Under this agenda item the conference considered the removal of a date limit to an allocation of the FSS within the frequency band 5091-5150 MHz, shared with the aeronautical radionavigation service (microwave landing systems) and the AM(R)S which supports AeroMACS, a WiMAX-based system for aeronautical safety communications at the airport surface.

The ICAO position was to support the removal of the date limit, while also asking for improved flexibility in the access to the frequency band by the aeronautical services, through an improvement in the flexibility to manage the allowed FSS satellite noise temperature increase by the aeronautical radionavigation and the $A M(R) S$ services operating in this frequency band). The outcome of the conference supported this position.

\section{WRC-15 Agenda Item 1.10 - Mobile Satellite Service in the 22-26 GHz Band}

This agenda item considered "spectrum requirements and possible additional spectrum allocations for the mobile-satellite service in the Earth-to-space and space-to-Earth directions, including the satellite component for broadband applications, including International Mobile Telecommunications (IMT), within the frequency range from $22 \mathrm{GHz}$ to $26 \mathrm{GHz} . .$. " The ICAO Position on this agenda item was to oppose any new mobile-satellite service allocation unless it had been demonstrated through agreed studies that there would be no impact on aviation use in the $24.25-24.65$ $\mathrm{GHz}$ bands in Regions 2 and 3. No new allocation was made by the conference in support of this agenda item.

\section{WRC-15 Agenda Item 1.11 - Earth Exploration Satellite Service primary allocation 7-8 GHz}

This agenda item considered " $a$ primary allocation for the Earth exploration-satellite service (Earth-to-space) in the $7-8 \mathrm{GHz}$ range..." The ICAO Position on this agenda item was to oppose any new allocation to the earth exploration satellite service (EESS) in the frequency band 8750-8850 $\mathrm{MHz}$, unless agreed studies showed no impact to aviation use. The conference allocated $7190-7250$ $\mathrm{MHz}$ under this agenda item, resulting in no impact on aviation.

WRC-15 Agenda Item 1.12 - Extension of worldwide allocation to the Earth Exploration Satellite Service

This agenda item considered "an extension of the current worldwide allocation to the Earth exploration-satellite (active) service in the frequency band $9300-9900 \mathrm{MHz}$ by up to $600 \mathrm{MHz}$ within the frequency bands $8700-9300 \mathrm{MHz}$ and/or 9900$10500 \mathrm{MHz} .$. " The ICAO Position on this agenda item was to oppose any new allocation to the EESS in the frequency band $9000-9200 \mathrm{MHz}$, as it has been demonstrated through agreed studies that EESS would impact aviation use and place constraints on the use of the frequency band by the aeronautical systems. WRC-15 agreed to a solution which included allocations to the EESS in the bands 92009300 and 9900-10400 MHz. These new allocations will not affect aeronautical services.

\section{WRC-15 Agenda Item 1.16 - Regulatory provisions and possible spectrum allocations for new Automatic Identification System}

This agenda item considered "regulatory provisions and spectrum allocations to enable possible new Automatic Identification System (AIS) technology applications and possible new applications to improve maritime radiocommunication..." The maritime automatic identification systems are fitted in search and rescue (SAR) aircraft to allow co-ordination of SAR activities in which both ships and aircraft are involved. Hence, the ICAO Position was to ensure that any change to the regulatory provisions and spectrum allocations resulting from this agenda item would not adversely impact the capability of SAR aircraft to effectively communicate with ships during disaster relief operations.

The conference developed a solution which facilitates the implementation of a VHF data exchange system (VDES) for ships and identified certain VHF channels in the maritime mobile service for VDES and other application specific messages. 
The solution protects existing AIS and is fully in line with the ICAO Position.

\section{WRC-15 Agenda Item 1.17 - Spectrum and regulatory requirements to support wireless avionics intra-communications (WAIC)}

This agenda item considered "possible spectrum requirements and regulatory actions, including appropriate aeronautical allocations, to support wireless avionics intra-communications (WAIC)..." The aerospace industry continues to develop new commercial aircraft to provide airlines and the flying public with more cost-efficient and environmentally friendly air transportation while maintaining required levels of safety and reliability. One important means of accomplishing these aims is to reduce overall aircraft weight, e.g. by reducing wiring, while providing multiple and redundant methods to transmit safety-related information within and on an aircraft. The utilization of wireless technologies is one potential method of accomplishing these goals thus providing environmental benefits such as reduction of carbon footprints as well as cost savings to manufacturers and operators.

In line with results of ICAO and ITU-R studies prior to the conference, the ICAO Position on this agenda item was to support a global $A M(R) S$ allocation in the radio altimeter frequency band of 4200-4400 MHz, exclusively reserved for WAIC systems operating in accordance with recognized international aeronautical standards.

The conference allocated the frequency band 4200-4400 MHz to the AM(R)S, exclusively reserved for WAIC, operating in accordance with international aeronautical standards. The associated Resolution requires that the WAIC systems protect the operation of the radio altimeters and operate in accordance with ICAO SARPs. It is expected that standardization work will be initiated within RTCA and/or EUROCAE before development of SARPs commences within ICAO.

\section{WRC-15 Agenda Item 1.18 - Radiolocation for automotive applications in the 77.5-78.0 GHz}

This agenda item considers " $a$ primary allocation to the radiolocation service for automotive applications in the $77.5-78.0 \mathrm{GHz}$ frequency band..." This agenda item was devised by WRC-12 to facilitate high resolution radar applications for the automotive industry. However this radar applications has also been proposed for aviation use.

As aircraft have become larger, the ability of the pilot to accurately taxi an aircraft around a busy airport has become more difficult and incidents of aircraft colliding with other objects on the airport have become more common. A potential solution to this issue would be to use off-the-shelf automotive radar located in the wing tips of aircraft to detect other ground objects that may be in the path of the taxiing aircraft. Hence the ICAO Position was to support the allocation of the frequency band 77.5 $78 \mathrm{GHz}$ to the radiolocation service in such a way as not to preclude its use on an advisory basis by taxiing aircraft.

Concerns by other users of this frequency band, namely the amateur, amateur satellite and the radio astronomy services were addressed by limiting a new allocation to "automotive and other ground based applications". While the new allocation is not conditional, the ITU-R has been tasked with further studies to assist administrations in ensuring compatibility between the incumbent services.

The new allocation made by the conference enables use of this frequency band for wing tip radars on aircraft, operational only while the aircraft is not airborne. This solution is fully in line with the ICAO Position.

\section{WRC-15 Agenda Item 9.1.1 - Protection of the MSS in 406-406.1 MHz}

This agenda item considers "protection of the systems operating in the mobile-satellite service in the band 406-406.1 MHz." Emergency locating transmitters (ELT) are an element of the COSPASSARSAT search and rescue satellite system. Mandatory carriage of ELTs for aircraft is specified in Annex 6 to the ICAO Convention and SARPs for ELTs are contained in Annex 10. The ICAO Position on this agenda item was to support increased protection of the COSPAS-SARSAT system in the frequency band $406-406.1 \mathrm{MHz}$.

The conference modified Resolution 205, addressing the protection of COSPAS-SARSAT. The modified provisions request Administrations not to make new frequency assignments in a guard band of $100 \mathrm{kHz}$ around the allocation of $406-406.1 \mathrm{MHz}$, 
used for COSPAS-SARSAT. This fully satisfies the concerns expressed in the ICAO Position.

\section{WRC-15 Agenda Item 9.1.6 - Definitions of Fixed Service, Fixed Station and Mobile Station}

This agenda item addressed "Studies towards review of the definitions of fixed service, fixed station and mobile station." The definitions in the Radio Regulations for fixed service, fixed station and mobile station are indirectly related to aeronautical services and hence any change of these definitions could have an impact on the interpretation of the definition of aeronautical mobile services. Hence the ICAO Position was to ensure that any change to the definitions would not adversely impact aviation. The conference did not make any modification to these definitions, an outcome fully in line with the ICAO Position.

\section{WRC-19 Agenda Items for Aviation}

WRC-15 Resolution 809 sets out the agenda for the 2019 WRC (WRC-19). As the study period progresses, many of the agenda items will be monitored for possible impact on civil aviation systems and spectrum, as unforeseen issues can emerge during the study cycle. However at the outset, several agenda items have been identified as being under the leadership of aviation or requiring active aviation participation [5]. The agenda items are:

- Agenda Item 1.10 - Global Aeronautical Distress and Safety System (GADSS)

o to consider spectrum needs and regulatory provisions for the introduction and use of the Global Aeronautical Distress and Safety System (GADSS)

- Agenda Item 1.11 - Globally harmonized railway bands

o to take necessary actions, as appropriate, to facilitate global or regional harmonized frequency bands to support railway

radiocommunication systems between train and trackside within existing mobile service allocations
- Agenda Item 1.12 - Globally harmonized Intelligent Transport Systems (ITS)

o to consider possible global or regional harmonized frequency bands, to the maximum extent possible, for the implementation of evolving Intelligent Transport Systems (ITS) under existing mobileservice allocations

- Agenda Item 1.16 - Wireless Access System/Radio Local Area Network (WAS/RLAN) in 5150-5925 MHz bands

o to consider issues related to wireless access systems, including radio local area networks (WAS/RLAN), in the frequency bands between $5150 \mathrm{MHz}$ and $5925 \mathrm{MHz}$, and take the appropriate regulatory actions, including additional spectrum allocations to the mobile service

- Agenda Item 9.14 - Space Planes

o to conduct studies to identify any required technical and operational measures, in relation to stations on board sub-orbital vehicles, that could assist in avoiding harmful interference between radiocommunication services.

In addition to these agenda items, other issues that will be addressed by ITU-R during the WRC-19 study cycle include: UAS C2 in the FSS (as noted above); Global Navigation Satellite System documentation; radar systems interference, compatibility and characteristics; and Global Flight Tracking and ADS-B reports.

\section{Conclusions}

The WRC-15 addressed a number of issues very relevant to civil aviation. Among those, four key WRC-15 agenda items were discussed in this paper, in terms of agenda item description and WRC-15 outcome. These items were: additional spectrum allocations for International Mobile Telecommunications; use of the FSS for beyond-lineof-sight command and control of Unmanned Aircraft Systems; FSS Earth Stations for Safe Operation of Aircraft, and Global Flight Tracking. 
In general, the conference outcomes are considered favorable for civil aviation. From the ICAO point of view, the results fully conformed to the ICAO Position. With the conclusion of WRC-15, it is noted that follow-up work must now be pursued in the implementation of the new WRC-15 resolutions, for example in the development of SARPs by ICAO's RPAS Panel. In addition, there are some on-going issues, in the case of WRC-15 Resolution 155 for UAS that will require additional work to bring the action initiated at WRC-15 to completion at WRC-19 and even at WRC-23.

Finally, WRC-15 has established the agenda for WRC-19. At least five WRC-19 agenda items will require active participation by aviation interests in order to insure positive outcomes for civil aviation. As well, several other agenda items must be monitored for possible emerging aviation impacts. Other issues related to civil aviation in the third category of aviation-related issues involving regulatory changes are expected to be addressed by ITU-R during the WRC-19 study cycle and must also be closely watched.

\section{References}

[1] ICAO FSMP WG/2 - Information Paper \# 01 - "Report on the results of the International Telecommunication Union (ITU) World Radiocommunication Conference (2015) (WRC-15) (2 to 27 November 2015, Geneva, Switzerland)”, February 2016.

[2] World Radiocommunicaiont Conference 2015 (http://www.itu.int/en/ITUR/conferences/wrc/2015/).

[3] Provisional Final Acts, World Radiocommunication Conference (WRC-15), ITU, 227 November, 2015.

[4] Final Acts of the Plenipotentiary Conference, ITU, Busan, 2014.

[5] ICAO FSMP WG/2 - Working Paper \# 01 “Aviation Issues for WRC-19, February 2016. 